\section{Concentrações de retinol e de beta-caroteno séricos e perfil nutricional de crianças em Teresina, Piauí, Brasil}

\section{Serum concentrations of retinol and beta-carotene, and nutritional status of children in Teresina, Piauí, Brazil}

Joilane Alves Pereira'

Adriana de Azevedo Paiva²

Denise Pimentel Bergamaschi ${ }^{3}$

Patrícia Helen de Carvalho Rondó ${ }^{1}$

Graciane Castro de Oliveira ${ }^{4}$

Iracelma Borges de Miranda Lopes ${ }^{4}$

Vanessa Kristinne Illison ${ }^{1}$

\section{Cecília Maria Resende Gonçalves-Carvalho ${ }^{4}$}

${ }^{1}$ Departamento de Nutrição, Faculdade de Saúde Pública, Universidade de São Paulo

${ }^{2}$ Núcleo de Estudos e Pesquisas Epidemiológicas, Universidade Estadual da Paraíba

${ }^{3}$ Departamento de Epidemiologia, Faculdade de Saúde Pública, Universidade de São Paulo

${ }^{4}$ Departamento de Nutrição, Universidade Federal do Piauí

Agradecimentos: Os nossos agradecimentos à Secretaria Municipal da Criança e do Adolescente Teresina, PI, pelo apoio no desenvolvimento da pesquisa; a Dra. Rachel Gitau, do "Institute of Child Health, University of London" e Dr. Alceu Jordão Júnior, da Faculdade de Medicina de Ribeirão Preto, Universidade de São Paulo-USP, São Paulo, pelo valioso suporte metodológico prestado; e à Fundação de Amparo à Pesquisa do Estado de São Paulo, pelo financiamento concedido (Processo no. 01/07899-1).

Correspondência: Adriana de Azevedo Paiva. Av. das Baraúnas no. 351, Prédio dos Mestrados, $2^{\circ}$ andar, Bairro Bodocongó, Campina Grande, Paraiba. CEP 58109-753. E-mail: aapaiva@yahoo.com.br
Resumo

Objetivo: Avaliar as concentrações séricas de retinol e beta-caroteno de pré-escolares em Teresina, Piauí, com caracterização do perfil antropométrico e do consumo alimentar. Material e Métodos: Estudo transversal envolvendo 135 crianças em creche municipal, com avaliação do estado nutricional pelos métodos: bioquímico (concentração sérica de retinol e betacaroteno), antropométrico (índices de peso para estatura - P/E e estatura para idade - E/I) e dietético (freqüência de consumo alimentar). Resultados: Observou-se prevalência de deficiência de vitamina A (DVA) de 8,9\% (IC95\%: 4,7 - 15,0\%) e existência de associação entre suplementação anterior e concentrações de retinol, com maior proporção de crianças com níveis normais de retinol entre as suplementadas ( $p=0,025)$. As concentrações de retinol e de beta-caroteno mostraram-se correlacionadas, porém com força leve a moderada ( $p \leq 0,021)$. Os percentuais de crianças com baixo $\mathrm{P} / \mathrm{E}$ e de baixa E/I foram de 1,9\% (IC95\%: 0,2 -6,8\%) e 9,7\% (IC95\%: 4,8 - 17,1\%), respectivamente. $\mathrm{Na}$ avaliação dietética verificou-se baixo consumo de alimentos ricos em vitamina A. Conclusões: A elevada prevalência de DVA nas crianças, combinada com a alta percentagem de crianças com valores aceitáveis de retinol, os baixos valores medianos de beta-caroteno, a alta percentagem de déficit estatural e a inadequação do consumo de alimentos ricos em vitamina $\mathrm{A}$, indicam a necessidade de se aprimorar as estratégias de educação em saúde e nutrição da população, incentivando o consumo de alimentos fontes de vitamina $\mathrm{A}$, como medidas auto-sustentáveis importantes no combate ao problema. Além disso, deve ser considerado o incentivo à fortificação dos alimentos e ao fortalecimento de Programas de suplementação.

Palavras-chave:Vitamina A. Beta-Caroteno. Pré-escolares. Estado Nutricional. 


\section{Abstract}

Objective: To evaluate serum concentrations of retinol and beta-carotene of children in Teresina, Piauí, Brazil, and to evaluate their anthropometric profile and consumption of food sources of vitamin A. Methods: Crosssectional study of 135 children from 36 to 83 months of age who attended a public child day care center. Nutritional status was evaluated by: biochemical (serum concentrations of retinol and beta-carotene), anthropometric (weight for height $-\mathrm{W} / \mathrm{H}$ and height for age $-\mathrm{H} / \mathrm{A}$ indexes), and dietary (frequency of consumption of food sources of vitamin A) methods. Results: The prevalence of vitamin A deficiency (VAD) was $8.9 \%$ (95\%CI: 4.7-15.0\%). An association between previous supplementation with vitamin $A$ and serum concentrations of retinol was observed, with a higher proportion of children with normal concentrations of retinol among those supplemented $(\mathrm{p}=0.025)$. There was a weak to moderate statistically significant correlation between the concentrations of retinol and beta-carotene, $(\mathrm{p} \leq 0.021)$. The prevalence of low W/H and low H/A was respectively 1.9\% (95\%CI: 0.2-6.8\%) and 9.7\% (95\%CI 4.8-17.1\%). The dietary evaluation showed low consumption of foods rich in vitamin A. Conclusions: The high prevalence of VAD combined to the prevalence of children with acceptable concentrations of retinol, low median concentrations of beta-carotene, low H/A, and inadequate ingestion of foods rich in vitamin $A$, reinforce the need to improve health and nutrition education in this population. Based on these results, we recommend the ingestion of foods rich in vitamin $\mathrm{A}$, as an important policy to deal with this nutritional problem. Efforts are also necessary to maintain food fortification and supplementation Programs.

Keywords: Vitamin A. Beta-Carotene. Preschool children. Nutritional Status.

\section{Introdução}

A vitamina A desempenha um papel importante na saúde. Baixos níveis séricos de vitamina A estão associados a maior mortalidade e morbidade, retardo de crescimento, aumento da suscetibilidade a infecções, xeroftalmia e cegueira noturna, sobretudo em crianças menores de 5 anos $^{1-3}$. A deficiência de vitamina A (DVA) é uma das carências nutricionais mais prevalentes no mundo subdesenvolvido, apresentando-se como um problema de Saúde Pública em mais de 70 países. Na América Latina, está muitas vezes associada à desnutrição protéico-energética (DPE), sendo que, no Brasil, a região Nordeste é considerada como a área onde o problema se apresenta em maior magnitude ${ }^{4-8}$.

A Organização Mundial da Saúde considera que a prevalência de baixas concentrações de retinol sérico $(<0,70 \mu \mathrm{mol} / \mathrm{l})$, quando igual ou maior a $20 \%$, caracteriza um problema grave de Saúde Pública ${ }^{9}$. Estudos epidemiológicos realizados no Nordeste brasileiro têm estimado elevada prevalência de baixas concentrações de retinol em pré-escolares, chegando a atingir cifras em torno de $55 \%$ no estado da Bahia ${ }^{10}$. Fernandes e col, $2005^{11}$, estudando préescolares de creches na cidade de Recife, observaram uma prevalência de DVA de $7 \%$, sugerindo uma redução dessa carência naquela cidade. Os autores atribuíram esse decréscimo às medidas emergenciais que vêm sendo adotadas no país, tais como a suplementação com megadoses de vitamina A em crianças dessa faixa etária. Além disso, ressaltam a existência de condições razoáveis de saúde e alimentação de crianças institucionalizadas ${ }^{4-8,10}$.

MacLaren \& Frigg, $2001^{12}$ apontam a idade, o estado fisiológico, a inadequação da ingestão alimentar, o desmame precoce, as doenças infecciosas, a desnutrição, a baixa condição socioeconômica e o sexo masculino como fatores que, de alguma forma, estão relacionados à etiopatogenia da DVA.

No Nordeste, observa-se a disponibilidade de muitos alimentos com elevado ou moderado conteúdo de vitamina $\mathrm{A}$, tanto 
de origem animal (fígado, leite e derivados) como de origem vegetal (manga, mamão, caju, goiaba, cenoura, abóbora, com destaque para os óleos de buriti e dendê, amplamente encontrados no nordeste brasileiro). Entretanto, estudo de consumo alimentar realizado em creches de Teresina, no Piauí, relata oferta inadequada de alimentos ricos em vitamina A e índices de adequação não atingindo $50 \%$ do recomendado ${ }^{7}$. Paiva $(2006)^{13}$, em estudo pioneiro envolvendo marcadores bioquímicos do status de vitamina A em pré-escolares na cidade de Teresina, sugeriu que a DVA apresenta-se como um problema moderado de Saúde Pública naquela população.

O presente estudo tem como objetivo avaliar as concentrações de retinol e de beta-caroteno séricos de crianças matriculadas em creche municipal de Teresina, Piauí, com caracterização do perfil antropométrico e do consumo alimentar.

\section{Metodologia}

Este estudo é parte de uma investigação realizada em 2003 na cidade de Teresina, Piauí, região Nordeste do Brasil, que teve como objetivo avaliar o impacto da suplementação com vitamina A no sistema imunológico de pré-escolares ${ }^{1}$.

O presente artigo apresenta os dados das crianças matriculadas em uma creche localizada em região periférica da cidade de Teresina, de um total de quatro incluídas no estudo original, uma vez que, por motivos operacionais, somente foram realizadas as análises de beta-caroteno para esta subamostra de crianças. Desta forma, neste estudo transversal foram envolvidas 135 crianças na faixa etária de 36 a 83 meses, no período de março a junho de 2003, considerando-se os seguintes critérios de inclusão: freqüência regular à creche, ausência de doenças crônicas e não utilização de suplementação com ferro e/ou vitamina A nos seis meses anteriores à coleta de dados. Além disso, não foram incluídas no estudo as crianças com processo infeccioso agudo clinicamente detectável.
A participação da criança foi voluntária e dependeu da presença da mãe ou responsável em reunião na qual foram expostos os objetivos e procedimentos da pesquisa, com preenchimento de termo de consentimento informado. Nesta reunião foi aplicado questionário para caracterização socioeconômica e demográfica.

O estudo foi aprovado pelos Comitês de Ética em Pesquisa da Universidade Federal do Piauí e da Faculdade de Saúde Pública da Universidade de São Paulo.

Foram aferidos, uma única vez, o peso e a estatura, pela manhã, com a criança em jejum, utilizando-se balança digital Soehnle ${ }^{\circledR}$ e antropômetro Microtoise ${ }^{\circledR}$, afixado em parede, sem rodapé. Seguiram-se, para a tomada das medidas antropométricas, procedimentos estabelecidos pela OMS $(1995)^{14}$. O estado nutricional foi avaliado segundo os índices antropométricos de estatura/idade (E/I) e peso/estatura (P/E). Consideraram-se desnutridas as crianças com valores de escores $\mathrm{Z}$ para E/I e P/E inferiores a -2 desvios padrão dos valores de referência do National Center for Health Statistics (NCHS, 1977) ${ }^{15}$. As crianças com valores de escore $\mathrm{Z}$ para $\mathrm{P} / \mathrm{E}>+2 \mathrm{DP}$ foram consideradas com sobrepeso.

Para avaliação do consumo alimentar foi aplicado aos responsáveis pelas crianças um questionário de freqüência do consumo alimentar (QFCA) contendo alimentos fontes de vitamina A (pré-formada e carotenóides precursores). O teor alimentar de vitamina A (equivalentes de retinol) por $100 \mathrm{~g}$ de alimento foi classificado em baixo $(<100 \mu \mathrm{g}$ ER), moderado (100-1199 $\mu \mathrm{g}$ ER) e alto $(\geq$ $1200 \mu \mathrm{g}$ ER). A freqüência de consumo foi classificada em diária, semanal (mais de 3 vezes ou menos de 3 vezes), mensal (1, 2 e 3 vezes), raramente ou nunca, de acordo com a proposta do International Vitamin A Consultative Group (1989) ${ }^{16}$.

Amostras de sangue ( $2 \mathrm{ml}$ ) foram colhidas das crianças, no início da manhã, em jejum, por punção venosa periférica, utilizando-se agulha e seringa descartáveis. Estas foram armazenadas em tubos secos, transparentes, envolvidos em papel alumínio, para evitar 
perdas em decorrência da exposição à luz. Após retração do coágulo e separação por centrifugação (400g por 10 minutos), as amostras foram acondicionadas em gelo seco e enviadas, por via aérea, para o Laboratório de Micronutrientes da Faculdade de Saúde Pública/USP, para determinação dos níveis de retinol e beta-caroteno séricos pelo método de cromatografia líquida de alta eficiência (HPLC), em equipamento SHIMADZU, com metodologia descrita por Erhardt et al. $(2002)^{17}$, com procedimento de calibração e repetibilidade. Esse método utiliza coluna e pré-coluna $\mathrm{C}_{18}$ de fase reversa e detector de UV-vis com lâmpada de deutério, com fase móvel composta por $80 \%$ acetonitrila, $3 \%$ metanol (100 Mm acetato de amônio, 0,1\% trietilamina) e 15\% dioxano. A fase móvel é bombeada pelo sistema por um fluxo de 1,5 $\mathrm{ml} / \mathrm{min}$ com pressões máxima de $350 \mathrm{Kgf} \mathrm{e}$ mínima de 0 Kgf. Os comprimentos de onda estabelecidos pelo método para o retinol e o beta-caroteno são, respectivamente, de 325 $\mathrm{nm}$ e $452 \mathrm{~nm}$; e o tempo de corrida ficou estabelecido em 4 minutos para o retinol e 22 minutos para o beta-caroteno.

Foram seguidas as recomendações da OMS para determinação dos pontos de corte para os níveis de retinol: deficiente: $<0,35$ $\mu \mathrm{mol} / \mathrm{L}$; baixo: 0,35 a 0,69 $\mu \mathrm{mol} / \mathrm{L}$; aceitável: 0,70 a $1,04 \mu \mathrm{mol} / \mathrm{L}$ e normal: $\geq 1,05 \mu \mathrm{mol} / \mathrm{L}$. A DVA é caracterizada quando os valores de retinol são $<0,70 \mu \mathrm{mol} / \mathrm{L}$.

A análise estatística incluiu apresentação dos dados em tabelas e gráficos; estimandose a prevalência de DVA e respectivo intervalo de confiança de $95 \%$, considerando-se a distribuição normal. Investigou-se a existência de associação entre DVA e as variáveis sexo, idade, suplementação anterior, cor da pele, escolaridade dos pais e renda per capita pelo teste Qui Quadrado de Pearson ou teste Exato de Fisher. A decisão estatística foi feita com base no valor descritivo do teste (valor de p). Para a investigação da relação entre nível de retinol e de beta-caroteno foi utilizado o coeficiente de correlação de Spearman.

Os dados foram digitados no programa Epi Info, versão $6.04 \mathrm{~b}^{18} \mathrm{e}$ analisados com auxílio do programa Stata $9.0^{19}$.
Os autores, através de documentação assinada e enviada a RBE, declaram não existir nenhum tipo de conflito de interesses.

\section{Resultados}

A população estudada $(n=135)$ apresentou idade média de 62,0 meses ( $\mathrm{DP}=13,5$ meses), sendo composta por $55 \%$ (74/135) de crianças do sexo masculino, $72 \%$ (97/135) classificadas como negras ou mulatas; $79 \%$ (97/123) com história de suplementação anterior com vitamina A (período superior a 6 meses antes do início da pesquisa); 78\% (104/133) declararam renda familiar per capita menor que $1 / 4$ do salário mínimo; $80 \%$ (106/133) das mães e 81\% (102/126) dos pais tinham como nível de educação, o ensino fundamental.

A concentração média de retinol observada foi de $1,27 \mu \mathrm{mol} / \mathrm{L}$ ( $\mathrm{n}=135 ; \mathrm{DP}=$ 0,47 $\mu \mathrm{mol} / \mathrm{L}$; IC95\%: 1,19 - 1,35 $\mu \mathrm{mol} / \mathrm{L}$ ), com prevalência de níveis inadequados de retinol sérico de 8,9\% (IC95\%: 4,7 - 15,0\%), indicando existência de problema de Saúde Pública leve. Observou-se, ainda, que 24,4\% das crianças apresentaram concentrações aceitáveis de retinol sérico $(0,70$ a 1,04 $\mu \mathrm{mol} / \mathrm{L}$ ) (Tabela 1).

Não foi verificada associação entre a prevalência de crianças com diferentes concentrações sangüíneas de retinol segundo as variáveis: sexo $(\mathrm{p}=0,920)$, idade $(p=0,284)$, cor da pele $(p=0,769)$, escolaridade da mãe ou do pai ( $\mathrm{p}=0,665 ; \mathrm{p}=$ 0,824 , respectivamente) e renda per capita $(p=0,879)$. Entretanto, observou-se associação entre a suplementação anterior e a concentração atual de retinol, verificandose uma maior proporção de crianças com concentrações normais entre aquelas suplementadas anteriormente $(\mathrm{p}=0,025)$ (Tabela 1, Gráfico 1).

A concentração mediana de beta-caroteno foi de $0,144 \mu \mathrm{mol} / \mathrm{L}$ (valor mínimo = 0,002 ; valor máximo $=1,147$ ), apresentandose inferior aos valores considerados adequados $(0,9-4,6 \mu \mathrm{mol} / \mathrm{L})$. Houve correlação significativa entre as concentrações de retinol e de beta-caroteno das crianças; porém 
Tabela 1 - Distribuição de crianças segundo variáveis sócio-demográficas e classificação da concentração de retinol sérico.

Table 1 - Distribution of children according to social and demographic factors and classification of serum concentrations of retinol.

\begin{tabular}{|c|c|c|c|c|c|c|}
\hline \multirow[t]{2}{*}{ Características } & \multicolumn{6}{|c|}{ Classificação da concentração de retinol (n, \%) } \\
\hline & Deficiente $^{(1)}$ & Baixo $^{(2)}$ & Aceitável $^{(3)}$ & Normal $^{(4)}$ & Total & Valor de $\mathrm{p}^{(5)}$ \\
\hline Total & $1(0,74)$ & $11(8,2)$ & $33(24,4)$ & $90(66,7)$ & $135(100)$ & \\
\hline \multicolumn{7}{|l|}{ Sexo } \\
\hline Masculino & $1(1,4)$ & $5(6,8)$ & $18(24,3)$ & $50(67,6)$ & $74(100)$ & \\
\hline Feminino & $0(-)$ & $6(9,8)$ & $15(24,6)$ & $40(65,6)$ & $61(100)$ & 0,920 \\
\hline \multicolumn{7}{|l|}{ Idade (meses) } \\
\hline $36 \mid--48$ & $0(-)$ & $4(16,0)$ & $6(24,0)$ & $15(60,0)$ & $25(100)$ & \\
\hline $48 \mid--60$ & $0(-)$ & $2(6,1)$ & $7(21,2)$ & $24(72,7)$ & $33(100)$ & \\
\hline $60 \mid--72$ & $0(-)$ & $3(9,7)$ & $4(12,9)$ & $24(77,4)$ & $31(100)$ & 0,284 \\
\hline $72 \mid--84$ & $1(2,2)$ & $2(4,4)$ & $16(34,8)$ & $27(58,7)$ & $46(100)$ & \\
\hline \multicolumn{7}{|l|}{ Suplementação anterior ${ }^{*}$} \\
\hline Sim & $1(1,0)$ & $9(9,3)$ & $18(18,6)$ & $69(71,1)$ & $97(100)$ & \\
\hline Não & $0(-)$ & $2(7,7)$ & $12(46,2)$ & $12(46,2)$ & $26(100)$ & 0,025 \\
\hline \multicolumn{7}{|l|}{ Cor } \\
\hline Branca & $0(-)$ & $4(10,5)$ & $8(21,1)$ & $26(68,4)$ & $38(100)$ & \\
\hline Não branca & $1(1,0)$ & $7(7,2)$ & $25(25,8)$ & $64(66,0)$ & $97(100)$ & 0,769 \\
\hline \multicolumn{7}{|l|}{ Escolaridade da mãe } \\
\hline Analfabeta/alfabetizada & $0(-)$ & $1(11,1)$ & $1(11,1)$ & $7(77,8)$ & $9(100)$ & \\
\hline Ensino Fundamental & $1(0,9)$ & $10(9,4)$ & $27(25,4)$ & $68(64,2)$ & $106(100)$ & 0,665 \\
\hline Ensino Médio & $0(-)$ & $0(-)$ & $4(22,2)$ & $14(77,8)$ & $18(100)$ & \\
\hline \multicolumn{7}{|l|}{ Escolaridade do pai } \\
\hline Analfabeto/alfabetizado & $0(-)$ & $2(14,3)$ & $4(28,6)$ & $8(57,1)$ & $14(100)$ & \\
\hline Ensino Fundamental & $1(1,0)$ & $9(8,8)$ & $24(23,5)$ & $68(66,7)$ & $102(100)$ & 0,824 \\
\hline Ensino Médio & $0(-)$ & $0(-)$ & $3(30,0)$ & $7(70,0)$ & $10(100)$ & \\
\hline \multicolumn{7}{|l|}{ Renda per capita $\left(\mathrm{SM}^{6}\right)$} \\
\hline 이-- 1/4 SM & $1(1,0)$ & $8(7,7)$ & $26(25,0)$ & $69(66,4)$ & $104(100)$ & 0,879 \\
\hline $1 / 4 \mid--1 / 2$ SM & $0(-)$ & $3(11,5)$ & $6(23,1)$ & $17(65,4)$ & $26(100)$ & \\
\hline $1 / 2 \mid--1^{3 / 5} S M$ & $0(-)$ & $0(-)$ & $0(-)$ & $3(100)$ & $3(100)$ & \\
\hline
\end{tabular}

esta relação apresentou-se de intensidade moderada nas crianças não suplementadas anteriormente (coeficiente de Spearman = $0,570 ; \mathrm{n}=26 ; \mathrm{p}=0,021)$ e leve nas crianças suplementadas (coeficiente de Spearman= 0,$273 ; \mathrm{n}=97 ; \mathrm{p}=0,020$ ) (Gráficos 1 e 2).

As prevalências de baixo peso para estatura e de baixa estatura para a idade foram iguais a $1,9 \%$ (IC95\%: 0,2 - 6,8\%) e 9,7\%
(IC95\%: 4,8-17,1\%), respectivamente. Não foi observada associação entre concentrações de retinol e beta-caroteno e os índices antropométricos ( $\mathrm{p}=0,366 ; \mathrm{p}=0,628$, respectivamente) (Tabela 2).

Observou-se baixo consumo de alimentos com alto ou moderado teores de vitamina A e alto consumo de alimentos com baixo teor desta vitamina (Tabela 3 ). 
Tabela 2 - Distribuição de crianças segundo índices antropométricos e classificação da concentração de retinol sérico. Table 2 - Distribution of children according to anthropometric index and classification of serum concentrations of retinol.

\begin{tabular}{|c|c|c|c|c|c|c|}
\hline \multirow[t]{2}{*}{ Características } & \multicolumn{6}{|c|}{ Classificação da concentração de retinol $(\mathrm{n}, \%)$} \\
\hline & Deficiente $^{(1)}$ & Baixo $^{(2)}$ & Aceitável $^{(3)}$ & Normal $^{(4)}$ & Total & Valor de $\mathrm{p}^{(5)}$ \\
\hline \multicolumn{7}{|c|}{ Escore $Z$ de estatura/idade } \\
\hline$\leq-2,00$ & $0(-)$ & $0(-)$ & $2(18,2)$ & $9(81,8)$ & $11(100)$ & \\
\hline$-2,01$ a 1,99 & $1(1,1)$ & $9(10,0)$ & $21(23,3)$ & $59(65,6)$ & $90(100)$ & \\
\hline$\geq 2,00$ & $0(-)$ & $0(-)$ & $1(50,0)$ & $1(50,0)$ & $2(100)$ & 0,671 \\
\hline \multicolumn{7}{|c|}{ Escore de peso/estatura } \\
\hline$\leq-2,00$ & $0(-)$ & $1(50,0)$ & $0(-)$ & $1(50,0)$ & $2(100)$ & \\
\hline$-2,01$ a 1,99 & $1(1,0)$ & $8(7,9)$ & $24(23,8)$ & $68(67,3)$ & $101(100)$ & 0,238 \\
\hline
\end{tabular}

(1) Deficiente: $<0,35 \mu \mathrm{mol} / \mathrm{Li} ;{ }^{(2)}$ Baixo: 0,35 a 0,69 $\mu \mathrm{mol} / \mathrm{L} ;{ }^{(3)}$ Aceitável:0,70 a 1,04 $\mu \mathrm{mol} / \mathrm{L} ; i^{(4)}$ normal: $\geq 1,05 \mu \mathrm{mol} / \mathrm{L} ;{ }^{(5)}$ Teste Exato de Fisher.

(1) Deficient: $<0.35 \mu \mathrm{mol} / \mathrm{L}$; ${ }^{(2)}$ Low:0.35 a $0.69 \mu \mathrm{mol} / \mathrm{L} ;{ }^{(3)}$ Acceptable: 0.70 a $1.04 \mu \mathrm{mol} / \mathrm{L}_{i}^{(4)}$ Normal: $\geq 1.05 \mu \mathrm{mol} / \mathrm{L} ;{ }^{\left({ }^{(5)}\right.}$ Fisher's Exact Test.

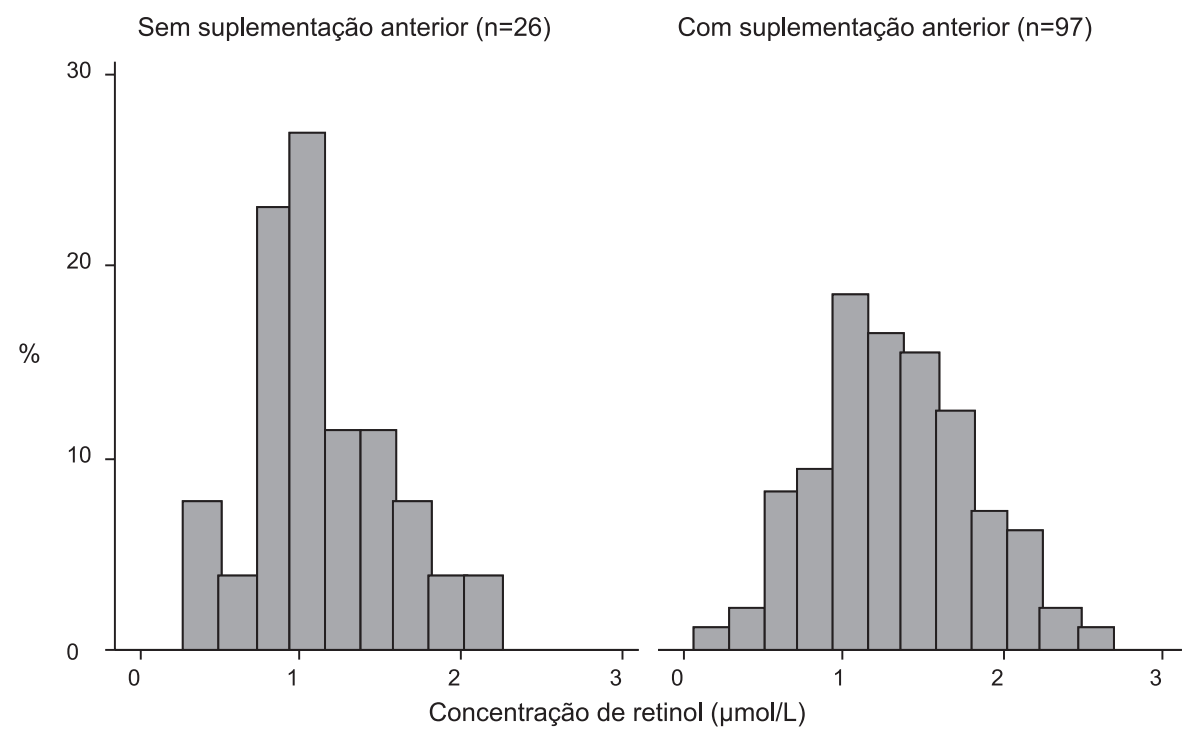

Gráfico 1 - Distribuição de crianças segundo concentrações de retinol e história de suplementação anterior com vitamina A até 6 meses antes do início do estudo.

Graph 1 - Distribution of children according to retinol concentrations and history of previous supplementation with vitamin A up to 6 months before the study.

\section{Discussão}

Os estudos realizados nos últimos anos no Nordeste do Brasil indicam que a DVA se apresenta como um problema de Saúde Pública em crianças da região, apesar do decréscimo nas taxas de prevalência observado em estudos mais recentes ${ }^{11,13}$. No presente estudo, a prevalência de $9 \%$ de DVA, encontrada em crianças institucionalizadas de 36 a 83 meses de idade, concorda com dados do estudo recente conduzido em Pernambuco por Fernandes e col. 2005 ${ }^{11}$ que estimaram uma prevalência de $7 \%$ (IC96\%:4,2 - 10,2\%) em pré-escolares de creches públicas da cidade de Recife.

O Piauí implementou o Programa Nacional de Suplementação de Vitamina A, proposto pelo Ministério da Saúde do Brasil ${ }^{20}$, que recomenda a administração periódica de doses terapêuticas de vitamina A para todas as crianças menores de cinco anos nas áreas de risco, somados a outros cuidados que integram as ações básicas de saúde. No presente estudo fica evidente a cobertura de tal Programa na cidade de Teresina, sendo 
Tabela 3 - Distribuição de crianças $(n=116)$ segundo consumo de alimentos fontes de vitamina A (em porcentagem). Table 3 - Distribution of children ( $n=116)$ according to consumption of food sources of vitamin A (in percentage).

\begin{tabular}{|c|c|c|c|c|c|c|}
\hline Teor/Alimento (100g) ${ }^{*}$ & Diariamente & 1 a 3 semanas & 4 a 5 semanas & 1 a 3 meses & 4 a 5 meses & Raramente \\
\hline \multicolumn{7}{|l|}{ ALTO } \\
\hline Fígado & - & 19,8 & - & 15,5 & - & 64,6 \\
\hline \multicolumn{7}{|l|}{ MODERADO } \\
\hline Batata doce & 1,7 & 38,8 & 3,5 & 8,6 & - & 47,4 \\
\hline Cenoura & 0,9 & 29,3 & 1,7 & 7,8 & - & 60,3 \\
\hline Manga & - & 7,8 & - & 6,0 & - & 86,2 \\
\hline Abóbora & 8,6 & 36,2 & 8,6 & 4,3 & - & 42,4 \\
\hline Queijo & 9,5 & 1,7 & - & 7,8 & 0,9 & 80,2 \\
\hline Pimentão & 38,8 & 11,2 & 2,6 & - & - & 47,4 \\
\hline Coentro & 65,5 & 4,3 & 0,9 & - & - & 29,3 \\
\hline Cheiro Verde & 64,7 & 4,3 & 0,9 & - & - & 30,2 \\
\hline \multicolumn{7}{|l|}{ BAIXO } \\
\hline Ovo de galinha & 3,5 & 75,0 & 9,5 & 5,2 & - & 6,9 \\
\hline Peixe & - & 48,3 & 3,5 & 18,1 & - & 30,2 \\
\hline Leite de vaca & 72,4 & 6,0 & 4,3 & 0,9 & 0,9 & 15,5 \\
\hline Farinha de mandioca & 32,8 & 27,6 & 9,5 & 2,6 & - & 27,6 \\
\hline Tomate & 43,1 & 35,3 & 8,6 & 1,7 & - & 11,2 \\
\hline Banana & 26,7 & 47,4 & 12,9 & 6,9 & - & 6,0 \\
\hline Laranja & 11,2 & 39,7 & 7,8 & 8,6 & - & 32,8 \\
\hline Abacate & 14,7 & 6,9 & - & - & - & 78,4 \\
\hline Melancia & 1,7 & 43,1 & 3,4 & 18,1 & - & 33,6 \\
\hline
\end{tabular}

* O teor alimentar de vitamina A (equivalentes de retinol) foi classificado em baixo (<100 $\mu \mathrm{g}$ ER), moderado (100-1199 $\mu \mathrm{g}$ ER) e alto ( $\geq 1200 \mu \mathrm{g}$ ER) para $100 \mathrm{~g}$ do alimento.

*Vitamin A content (retinol equivalents) was classified ( $\mu \mathrm{g}$ ER) as low $(<100 \mu \mathrm{g}$ ER), moderate (100-1199 $\mu \mathrm{g}$ ER) and high (>1200 $\mu \mathrm{g}$ ER) per $100 \mathrm{~g}$ of food.

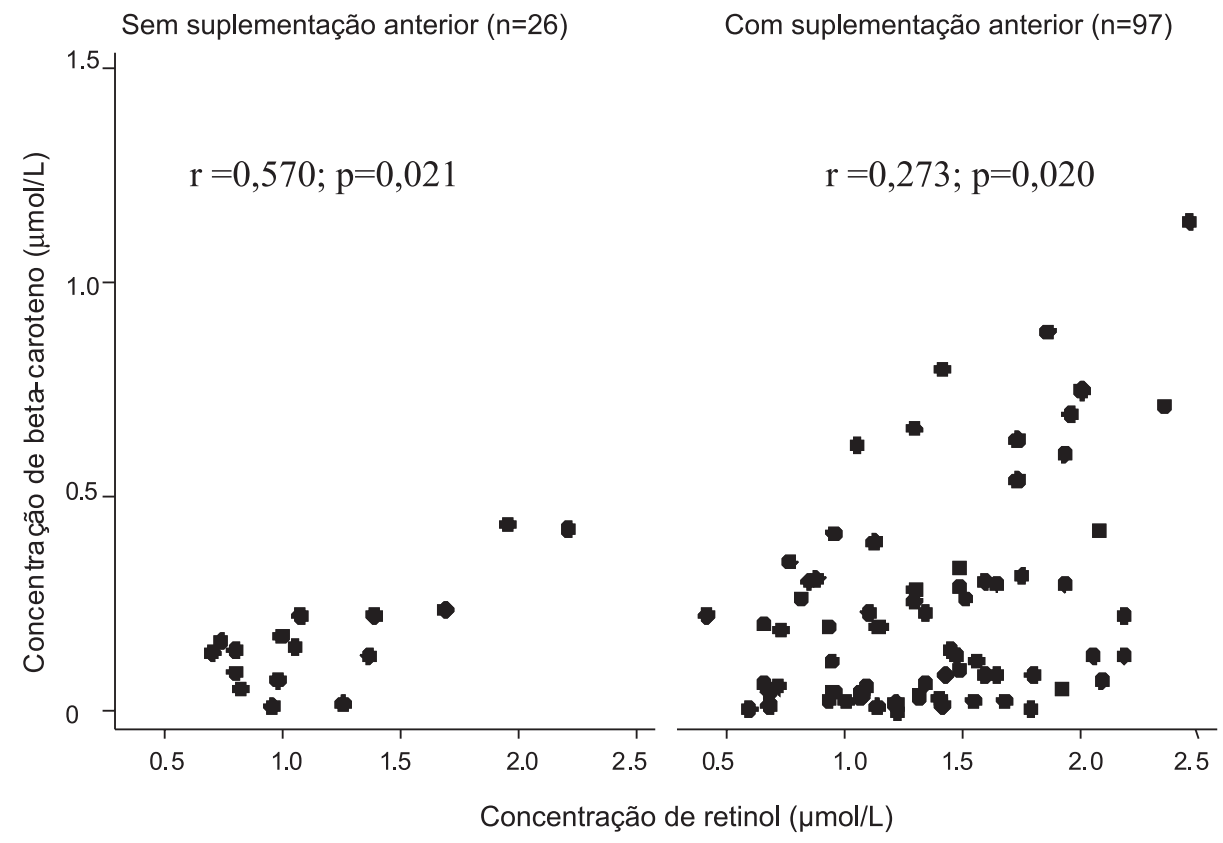

Gráfico 2 - Distribuição de crianças segundo concentrações de retinol e de $\beta$-caroteno.

Graph 2 - Distribution of children according to retinol concentrations and $\beta$-carotene. 
que perto de $80 \%$ das crianças já haviam recebido megadoses de vitamina A pelo menos uma vez desde o nascimento.

Além da existência deste Programa, deve-se considerar que as crianças estudadas podem apresentar situação de saúde diferenciada por serem institucionalizadas, repercutindo de um modo geral no estado nutricional, inclusive nas concentrações séricas de retinol.

A relação entre sexo da criança e deficiência de vitamina A é controversa, estando presentes, na literatura internacional, estudos que descrevem uma tendência de crianças do sexo masculino apresentarem maior risco de desenvolver DVA, quando comparadas a crianças do sexo feminino ${ }^{21}$. Essa relação não foi observada neste estudo, bem como em outros realizados no Nordeste do Brasil ${ }^{5,22}$.

A não identificação de existência de relação entre concentrações séricas de retinol e idade, cor da pele, escolaridade da mãe e do pai, renda per capita e situação antropométrica foram também descritas em estudo recente ${ }^{11}$. É provável que a grande homogeneidade apresentada pela população aqui estudada tenha dificultado a identificação de associações estatísticas.

A observação de associação estatística entre suplementação anterior e valores atuais de retinol sérico indica o efeito das ações do Programa oficial de suplementação. Podese inferir que a redução do quadro carencial seja reflexo de medidas emergenciais que vêm sendo tomadas na região desde a década de $80^{23,24}$.Vale lembrar que nenhuma criança incluída nesse estudo havia sido suplementada por um período inferior a 6 meses.

A estratégia de suplementação com altas doses de vitamina A se fundamenta no fato de que grande quantidade pode ser armazenada no fígado para ser utilizada quando for necessário e que megadoses de 200000 UI em crianças de 6 a 11 meses de idade, a cada 4 a 6 meses, exerce uma ação eficaz e de custo moderado contra a DVA e suas seqüelas ${ }^{25}$.

O estado nutricional avaliado pelos índices antropométricos indicou baixa pre- valência de baixo peso para estatura $(\mathrm{P} / \mathrm{E}$ : 1,9\%; IC95\%: 0,2-6,8\%) e considerável prevalência de retardo do crescimento linear (E/I: 9,7\%; IC95\%: 4,8 - 17,1\%), com valores muito próximos do encontrado em outros inquéritos realizados na região Nordeste ${ }^{26}$. Esta situação indica necessidade de monitorar o crescimento nos primeiros anos de vida por meio de um controle mais rigoroso do estado nutricional.

A redução de déficits de crescimento tem sido observada nas últimas décadas, para a maioria dos países em desenvolvimento ${ }^{27}$. Porém, apesar da evidente melhora no estado nutricional das crianças, na região Nordeste, ainda se observa percentual maior de desnutrição, principalmente quando comparada à região Sudeste do país ${ }^{28}$.

O hábito alimentar inadequado, se considerados alimentos fonte de vitamina A, pode ser identificado ao se verificar que apenas $20 \%$ dos pré-escolares relatam o consumo de alimentos com alto teor de vitamina A com consumo de 1-3 vezes por semana e $65,0 \%$ referem raramente ou nunca consumirem este alimento. Verifica-se, por outro lado, que os alimentos que compõem a lista de baixo conteúdo em vitamina A foram os mais consumidos diariamente. Acreditamos que os baixos valores medianos de beta-caroteno observados neste estudo, assim como em outros estudos da literatura internacional e nacional, sejam decorrentes da baixa ingestão dietética recente de alimentos fonte deste micronutriente ${ }^{29,30}$ Ao contrário, os valores de retinol não teriam sido determinados em função da inadequação alimentar observada, uma vez que nesta população a prática de suplementação anterior foi bastante prevalente.

A seleção de alimentos a serem consumidos é um processo complexo e pode ser influenciado por muitos outros fatores além do acesso aos alimentos e o conhecimento de nutrição, como, exemplo, fatores culturais e hábitos alimentares da população. Este conjunto de situações indica a importância da educação nutricional e seu relevante papel no estabelecimento de hábitos alimentares adequados como 
parte das ações de controle de deficiências nutricionais na região.

O estudo mostra que a DVA representou um problema de Saúde Pública do tipo leve nas crianças institucionalizadas na faixa etária de 36 a 83 meses de idade consideradas em nosso estudo, com prevalência de 9\% de concentrações séricas inadequadas de retinol. Acrescente-se ao problema o elevado percentual de crianças com valores aceitáveis de retinol e os baixos valores me- dianos de beta-caroteno, além da ocorrência elevada de déficit de crescimento linear e inadequação de consumo de alimentos ricos em vitamina A. Esses achados indicam a necessidade de educar a população e incentivar o consumo de alimentos fontes de vitamina A e seus precursores, como medidas auto-sustentáveis importantes no controle e combate à DVA, complementadas pelo incentivo à fortificação dos alimentos e à adoção de Programas de suplementação.

\section{Referências}

1. Paiva AA, Rondó PHC, Oliveira CAF, Vaz-de-Lima LRA, Ueda M, Carvalho CMRG, Bergamaschi DP. The impact of vitamin A supplementation on the cellular immune system of preschool children. In: Vorster HH, Blaavw R, Dhansay MA, Kuzwayo PMN, Moeng L, Wentzel-Viljoen E, guest editors. Proceedings of the 18th International Congress of Nutrition 2005; South Africa; 2005. ISBN 3-8055-8015-0. 13p.

2. Villamor E, Fawzi WW. Effects of vitamin A supplementation on immune responses and correlation with clinical outcomes. Clin Microbiol Rev 2005; 18: 446-64.

3. FAO/WHO. International Conference on Nutrition Strategies (Ed). FAO, Rome, Italy, 1992, p.35.

4. El Beitune P, Duarte G, Quintana SM, Figueiró-Filho EA, Vannucchi H. Hipovitaminose A: cofator clínico deletério para o homem. Medicina, 2003; 36: 5-15.

5. Martins MC, Santos LMP, Assis AMO. Prevalência de hipovitaminose A em pré-escolares no estado de Sergipe, 1998. Rev Saúde Pública 2004; 38(4): 537-42.

6. Prado MS, Assis AMO, Martins MC, Nazaré MPA, Rezende IFB, Conceição MEP. Hipovitaminose A em crianças de áreas rurais do semi-árido baiano. Rev Saúde Pública 1995; 29(4): 295-300.

7. Carvalho CMG, Farfan BCW, Venconsky R. Prevalência de hipovitaminose A em crianças da periferia do município de São Paulo, Brasil.Cad Saúde Pública 1995; 11: 85-96.

8. Araújo KC, Carvalho CMRG, Paz SMRS. Avaliação do consumo alimentar de vitamina A de crianças assistidas em creches comunitárias, Teresina(PI), Brasil. NutrireRev Soc Bras Alim Nutr 2001; 22: 7-19.

9. World Health Organization. Indicators for assessing vitamin A deficiency and their application in monitoring and evaluating intervention programmes. Geneva: WHO; 1996.
10. Santos LMP, Assis AMO, Martins MC, Araújo MPN, Morris SS, Barreto ML. Situação nutricional e alimentar de pré-escolares no semi-árido da Bahia (Brasil): II Hipovitaminose A. Rev Saúde Públ 1996; 30: 67-74.

11. Fernandes TFS, Diniz AS, Cabral PC, Oliveira RS, Lola MMF, Silva SMM et al. Hipovitaminose A em préescolares de creches públicas do Recife: indicadores bioquímico e dietético. Rev Nutr 2005; 18(4): 471-80.

12. McLaren DS, Frigg M. Sight and life manual on vitamin A deficiency disorders (VADD). 2 ed. Switzerland: Task Force Sight and Life; 2001.

13. Paiva AA, Rondó PHC, Gonçalves-Carvalho CMR, Illison VK, Pereira JA, Lima LRAV, Oliveira CA, Ueda M, Bergamaschi DP. Prevalência de deficiência de vitamina A e fatores associados em pré-escolares de Teresina, Piauí, Brasil. Cad Saúde Pública 2006; 22 (9): 1979-87.

14. Organização Mundial da Saúde. El estado físico: uso e interpretación de la antropometria. Série de Informes Técnicos 854. Genebra: WHO; 1995.

15. NCHS - National Center for Health Statistics. Growth curves for children, birth to 18 years, United States. Vital Health Stat 1977; 11(165): 1-74.

16. IVACG. Guidelines for the development of a simplified assessment to identify groups at risk for inadequate intake of vitamin A. Report of IVACG, Washington; 1989.

17. Erhardt JG, Mack H, Sobeck U, Biesalski HK. $\beta$-Carotene and -tocopherol concentration and antioxidant status in buccal mucosal cells and plasma after oral supplementation. Br J Nutr 2002; 87: 471-5.

18. Dean AG. Epi Info [computer program]. Version 6: a word-processing, database, and statistics program for epidemiology on microcomputers. Atlanta, Georgia: Centers of Disease Control and Prevention; 1994.

19. Stata. Statistical Software. College Station. Texas: Stata Corporation; 2005. 
20. Ministério da Saúde. Secretaria de Atenção à Saúde. Departamento de Atenção Básica. Vitamina A Mais: Programa Nacional de Suplementação de Vitamina A: Condutas Gerais. Série A. Normas e manuais técnicos. Brasília: Ministério da Saúde; 2004.

21. Bloem MW, Wedel M, Egger R, Speek AJ, Shrisjver J. Iron metabolism and vitamin A deficiency in children in the Northwest Thailand. Am J Clin Nutr 1989; 50: 332-338

22. Andrade SLLS. Prevalência de hipovitaminose Ae potenciais fatores de risco em crianças menores de 5 anos no Estado de Pernambuco 1997 [tese de doutorado]. Recife: Universidade Federal de Pernambuco; 1997.

23. Varela RM, Teixeira SG, Batista M. Hipovitaminose A in the sugarcane zone of the Southern Pernambuco State, Northeast Brazil. Am J Clin Nutr 1972; 28(8): 800-04.

24. Flores H, Araújo CR. Liver levels of retinol in unselected necropsy specimens: a prevalence survey of vitamin A deficiency in Recife, Brazil. Am J Clin Nutr 1984; 40: $146-52$.

25. Sommer A. Vitamin A deficiency and its consequences: a field guide to detection and control. In: Epidemiology. 3 ed. Geneva: WHO; 1995.
26. Diniz AS. Aspectos clínicos, subclínicos e epidemiológicos da hipovitaminose A no Estado da Paraíba [tese de doutorado]. Recife: Universidade Federal de Pernambuco; 1997.

27. Monteiro CA, Benício MHD’A, Freitas ICM. Melhoria em indicadores de saúde associados à pobreza no Brasil nos anos 90: descrição, causas e impacto sobre desigualdades regionais. São Paulo: NEPENS; 1997.

28. Ramalho RA, Anjos LA, Flores H. Valores séricos de vitamina A e teste terapêutico em pré-escolares atendidos em uma Unidade de Saúde do Rio de Janeiro, Brasil. Rev Nutr 2001; 14: 5-12.

29. Olson JA. Serum levels of vitamin A and carotenoids as reflectors of nutritional status. J Natl Câncer Inst 1984; 73: 1439-44.

30. Ferraz IS, Daneluzzi JC, Vannucchi H, Jordão AA Jr, Ricco RG, Del Ciampo LA et al. Detection of vitamin A deficiency in Brazilian preschool children using the serum 30-day dose-response test. Eur J Clin Nutr 2004; 58(10): 1372-7.

Recebido em: 21/08/07 Versão final reapresentada em: 20/02/08 Aprovado em: 19/03/08 